DOI 10.4171/JEMS/344

Miklós Abért · Nikolay Nikolov

\title{
Rank gradient, cost of groups and the rank versus Heegaard genus problem
}

Received August 12, 2008 and in revised form July 8, 2010

\begin{abstract}
We study the growth of the rank of subgroups of finite index in residually finite groups, by relating it to the notion of cost. As a by-product, we show that the 'rank vs. Heegaard genus' conjecture on hyperbolic 3-manifolds is incompatible with the 'fixed price problem' in topological dynamics.
\end{abstract}

\section{Introduction}

Let $\Gamma$ be a finitely generated group. A chain in $\Gamma$ is a sequence $\Gamma=\Gamma_{0} \geq \Gamma_{1} \geq \cdots$ of subgroups of finite index in $\Gamma$. Let $T=T\left(\Gamma,\left(\Gamma_{n}\right)\right)$ denote the coset tree of the chain, a rooted tree on the set of right cosets of the subgroups $\Gamma_{n}$ with edges $\left(\Gamma_{n} g, \Gamma_{n+1} g\right)$ for all $g \in \Gamma$ and $n \in \mathbb{N}$. The boundary $\partial T$ of $T$ is the set of infinite rays starting from the root; it is naturally endowed with the product topology and product measure coming from the tree. The group $\Gamma$ acts by automorphisms on $T$; this action extends to measure preserving homeomorphisms of the boundary.

We say that a chain $\left(\Gamma_{n}\right)$ is Farber if the action of $\Gamma$ on the boundary of its coset tree $T=T\left(\Gamma,\left(\Gamma_{n}\right)\right)$ is essentially free, that is, almost every element of $\partial T$ has trivial stabilizer in $\Gamma$. This is the case for example when the chain consists of normal subgroups of $\Gamma$ and their intersection is trivial. Note that $\partial T$ then is simply the profinite completion of $\Gamma$ with respect to $\left(\Gamma_{n}\right)$ endowed with the normalized Haar measure.

For a group $G$ let $d(G)$ denote the minimal number of generators (or rank) of $G$. Let the rank gradient of $\Gamma$ with respect to $\left(\Gamma_{n}\right)$ be defined as

$$
\operatorname{RG}\left(\Gamma,\left(\Gamma_{n}\right)\right)=\lim _{n \rightarrow \infty} \frac{d\left(\Gamma_{n}\right)-1}{\left|\Gamma: \Gamma_{n}\right|} .
$$

This notion has been introduced by Lackenby [17].

Our first theorem relates the rank gradient of a Farber chain to the cost of the action of the group on the boundary of the coset tree. The analytic notion of cost was introduced by Levitt [18] and used by Gaboriau [11] to show that free groups of different rank do not admit orbit equivalent measurable actions (see also the book of Kechris and Miller [15]).

M. Abert: Alfréd Rényi Institute of Mathematics, Reáltanoda utca 13-15, H-1053 Budapest, Hungary; e-mail: abert@ renyi.hu

N. Nikolov: Department of Mathematics, Imperial College London, London, SW7 2AZ, UK; e-mail:nnikolov@imperial.ac.uk 
Theorem 1. Let $\left(\Gamma_{n}\right)$ be a Farber chain in $\Gamma$. Then

$$
\operatorname{RG}\left(\Gamma,\left(\Gamma_{n}\right)\right)=\operatorname{cost}(E)-1
$$

where $E$ denotes the orbit relation given by the action of $\Gamma$ on the boundary of the coset tree $T\left(\Gamma,\left(\Gamma_{n}\right)\right)$.

Theorem 1 allows us to clash two well-known problems, one in 3-manifold theory and the other in topological dynamics.

Rank vs Heegaard genus conjecture. Let $M$ be a compact, orientable, hyperbolic 3manifold. Then the Heegaard genus of $M$ equals the rank of the fundamental group of $M$.

It is easy to see that the Heegaard genus is always greater than or equal to the rank. The problem dates back to Waldhausen [32], who asked it for arbitrary 3-manifolds. This was proved false for Seifert manifolds by Boileau and Zieschang in [7] (see also [26]), but it remains open for hyperbolic 3-manifolds. For the above formulation, see [27, Conjecture 1.1]. Also, it is not known whether the ratio of the two quantities can become arbitrarily large even for arbitrary 3-manifolds; the best known lower bound comes from the Boileau-Zieschang result.

A countable group $\Gamma$ has fixed price if every essentially free measure-preserving Borel action of $\Gamma$ has the same cost. Gaboriau [11] established fixed price for a large class of groups, including free groups, higher rank nonuniform real irreducible lattices and groups containing an infinite amenable normal subgroup and asked whether the following holds.

Fixed price problem. Does every countable group have fixed price?

Theorem 2. Either the rank vs Heegaard genus conjecture is false or the fixed price problem has a negative solution.

Moreover, if the fixed price problem has an affirmative answer, then the rank vs Heegaard genus conjecture fails in the following strong senses. First, the ratio of the Heegaard genus and the rank of the fundamental group of a compact, orientable hyperbolic 3-manifold can get arbitrarily large. Second, the counterexamples are not exotic, rather this seems to be the general asymptotic behaviour of arithmetic hyperbolic 3-manifolds.

The contradiction between the two problems is established via the (unknown) answer to the following question: Does the rank gradient $\operatorname{RG}\left(\Gamma,\left(\Gamma_{n}\right)\right)$ depend on the choice of the Farber chain $\left(\Gamma_{n}\right)$ in $\Gamma$ ?

If it does, then Theorem 1 trivially provides a negative answer to the fixed price problem. In the other direction, there are specific (uniform or nonuniform) arithmetic lattices in $\mathrm{SL}_{2}(\mathbb{C})$ (e.g. $\mathrm{SL}_{2}(\mathbb{Z}[i])$ ) that viewed as abstract groups, possess a chain of subgroups with vanishing rank gradient. On the other hand, being arithmetic groups, they have property $(\tau)$ with respect to congruence subgroups. Using work of Lackenby [17], this allows one to construct a covering tower of the 3-manifold corresponding to the lattice, where the Heegaard genus grows linearly. Now, if the rank gradient is independent of the chain, the rank must grow sublinearly on this tower, making the ratio of the Heegaard genus and the rank arbitrarily large. 
We shall derive the independence of the rank gradient from the chain from a hypothesis that is much weaker than fixed price (see [15, Problem 25.5]).

Multiplicativity of cost-minus-1 problem. Let $\Gamma$ be a measurable, essentially free action on $(X, \mu)$ and let $H$ be a subgroup of $\Gamma$ of finite index. Is it true that

$$
\operatorname{cost}(H, X)-1=(\operatorname{cost}(\Gamma, X)-1)|\Gamma: H| ?
$$

That is, does the cost of an action behave the same way as the rank for free groups?

The whole theory developed in this paper has a close connection to $L^{2}$ Betti numbers. The Lück approximation result [20] implies that for finitely presented groups and normal chains with trivial intersection, if we replace $d\left(\Gamma_{n}\right)$ with the first Betti number $\beta_{1}\left(\Gamma_{n}\right)$ in the definition of rank gradient, then the limit equals the first $L^{2}$ Betti number $\beta_{1}^{2}(\Gamma)$. This has been generalized by Farber [9] to chains satisfying his condition (see also [6] for examples showing the necessity of this condition). On the other hand, Gaboriau [10] has introduced $L^{2}$ Betti numbers of a measurable equivalence relation $E$ and asked whether $\beta_{1}^{2}(E)=\operatorname{cost}(E)-1$ in general. An affirmative answer to Gaboriau's question would imply the surprising result that the asymptotic growth of $\beta_{1}\left(\Gamma_{n}\right)$ and $d\left(\Gamma_{n}\right)$ are equal for all finitely presented residually finite groups. Note that without the condition of finite presentability this is false as shown in recent results of D. Osin [22] and J.-C. SchlagePuchta [25]. This is expected, as for this more general class, Lück approximation does not imply anything on the behaviour of $\beta_{1}$.

Theorem 1 immediately allows us to compute the rank gradient for a class of groups where elementary methods seem to fail working.

Theorem 3. Let $\Gamma$ be a residually finite group with an infinite amenable normal subgroup and let $\Gamma_{n}$ be a Farber chain in $\Gamma$. Then $\operatorname{RG}\left(\Gamma,\left(\Gamma_{n}\right)\right)=0$.

This generalizes a result of Lackenby [17] who proved the result for finitely presented amenable groups.

We also answer a question of Kechris and Miller [15, Problem 35.7]. They asked whether for a countable infinite Euclidean domain $D$ the group $\operatorname{SL}(2, D)$ has cost 1 if and only if $D$ has infinitely many units. The answer is negative: as we shall see, $\operatorname{SL}(2, \mathbb{Z}[i])$ has cost 1 and $\mathbb{Z}[i]$ has finitely many units. A well-known conjecture by Thurston asserts that every hyperbolic 3-manifold virtually fibres over the circle. This would imply that any lattice in $\operatorname{SL}(2, \mathbb{C})$ has cost 1 . On the other hand, as we show in Section 5, lattices in $\operatorname{SL}(2, \mathbb{R})$ have positive rank gradient with respect to any Farber chain.

Note that in a recent paper [14] Kechris has applied our Theorem 1 to present an alternative proof that free groups of rank $n$ have fixed price $n$.

The paper is organized as follows. In Section 2 we define measurable actions, graphings and the cost. We also introduce a new type of graphing and an invariant, the groupoid cost. We relate the groupoid cost to the cost and prove a general approximation result on graphings. In Section 3 we apply this result to subgroup chains and express the groupoid cost in terms of the rank gradient, thus proving Theorem 1. In Section 4 we use the theory developed so far to prove Corollary 3 and compute the rank gradient for some important classes of groups. We also discuss what happens if we relax the Farber condition on the 
chain. Finally, in Section 5 we consider the rank gradient of lattices in Lie groups, prove Theorem 2 and answer the question of Kechris and Miller.

\section{Graphings and cost}

In this section we introduce Borel actions, the standard notion of cost, as well as a new version of cost that will be useful in proving Theorem 1 .

Let $X$ be a standard Borel space. Let the countable group $\Gamma$ act on $X$ by Borel automorphisms. Let $\mu$ be a $\Gamma$-invariant probability measure on $X$. We assume that the $\Gamma$-action has finitely many ergodic components.

Let us define the relation $E$ on $X$ by

$$
x E y \quad \text { if there exists } \gamma \in \Gamma \text { with } y=x \cdot \gamma \text {. }
$$

Then $E$ is a Borel equivalence relation and every equivalence class is countable.

Since $E$ is a subset of $X \times X$, it can also be considered as a graph on $X$. A Borel subgraph of $E$ is a directed graph on $X$ such that the edge set is a Borel subset of $E$.

Let $S \subseteq X \times X$ be an arbitrary graph on $X$. A path from $x$ to $y$ in $S$ of length $k \geq 1$ is a sequence $x_{0}, x_{1}, \ldots, x_{k} \in X$ such that:

- $x_{0}=x, x_{k}=y$;

- $\left(x_{i}, x_{i+1}\right) \in S$ or $\left(x_{i+1}, x_{i}\right) \in S(0 \leq i \leq k-1)$.

Note that this defines an undirected path in $S$. For $k \geq 1$ let us define the graph $S^{k}$ by

$$
(x, y) \in S^{k} \quad \text { if } \quad x=y \text { or there is a path from } x \text { to } y \text { in } S \text { of length at most } k \text {. }
$$

We say that a subgraph $S$ of $E$ spans $E$ if for any $(x, y) \in E$ with $x \neq y$ there exists a path from $x$ to $y$ in $S$. Trivially, this holds if and only if

$$
\bigcup_{n} S^{n}=E
$$

The edge-measure of a Borel subgraph $S$ of $E$ is defined as

$$
e(S)=\int_{x \in X} \operatorname{deg}_{S}(x) d \mu
$$

where $\operatorname{deg}(x)$ is the number of edges in $S$ with initial vertex $x$ :

$$
\operatorname{deg}_{S} x=|\{y \in X \mid(x, y) \in S\}| .
$$

Note that $e(S)$ may be infinite. The cost of $E$ is defined as

$$
\operatorname{cost}(E)=\operatorname{cost}(\Gamma, X)=\inf e(S)
$$

where the infimum is taken over all Borel subgraphs $S$ of $E$ that span $E$. The cost of $\Gamma$ is defined as

$$
\operatorname{cost}(\Gamma)=\inf \operatorname{cost}(\Gamma, X)
$$


where the infimum is taken over all ergodic, essentially free actions of $\Gamma$ on a standard Borel space $X$.

We say that $\Gamma$ has fixed price $c$ if all ergodic, essentially free actions of $\Gamma$ on a standard Borel space $X$ have cost $c$. It is not known whether every countable group has fixed price [11].

If $\Gamma$ is generated by $g_{1}, \ldots, g_{d}$ then it is easy to see that the set

$$
\bigcup_{i=1}^{d} \bigcup_{x \in X}\left\{\left(x, x \cdot g_{i}\right)\right\}
$$

is a spanning Borel subrelation of $E$ of edge measure $d$. This implies the following.

Lemma 4. $\operatorname{cost}(\Gamma, X) \leq d(\Gamma)$.

Now we will look at cost from another point of view, using graphings. This notion basically comes from [18] but we will need a modification of it for use in a more general setting. The advantage is that this notion will work for non-essentially-free (even finite) actions as well.

Let us consider the product space $X \times \Gamma$ where $\Gamma$ is endowed with the discrete topology and the counting measure. Denote the product measure by $e$. A graphing is a Borel subset of $X \times \Gamma$. For a graphing $M$ and $\gamma \in \Gamma$ let

$$
M_{\gamma}=\{x \in X \mid(x, \gamma) \in M\}
$$

be the $\gamma$-fibre of $M$. For $x \in X$ let

$$
N(M, x)=\left\{\gamma \in \Gamma \mid x \in M_{\gamma}\right\} \quad \text { and } \quad \operatorname{deg}_{M} x=|N(M, x)|
$$

be the set of neighbours and the degree of $x$ in $M$. Using this notation, we have

$$
e(M)=\sum_{\gamma \in \Gamma} \mu\left(M_{\gamma}\right)=\int_{x \in X} \operatorname{deg}_{M}(x) d \mu .
$$

We will need a definition of powering of graphings. Let $I$ be the graphing defined by

$$
I_{\gamma}= \begin{cases}X, & \gamma=1 \\ \varnothing, & \text { otherwise }\end{cases}
$$

For a graphing $M$ let the graphing $M^{\top}$ be defined by

$$
M_{\gamma}^{\top}=M_{\gamma^{-1}} \cdot \gamma^{-1} \quad(\gamma \in \Gamma)
$$

and let $\bar{M}$ be defined by

$$
\bar{M}=M \cup M^{\top} \cup I .
$$

For graphings $M$ and $N$ let us define the graphing $M \cdot N$ by

$$
(M \cdot N)_{\gamma}=\bigcup_{\delta \in \Gamma}\left(M_{\delta} \cap\left(N_{\delta^{-1} \gamma} \cdot \delta^{-1}\right)\right) \quad(\gamma \in \Gamma),
$$


that is,

$(x, \gamma) \in M \cdot N \leftrightarrow \exists \gamma_{1}, \gamma_{2} \in \Gamma$ with $\left(x, \gamma_{1}\right) \in M,\left(x \gamma_{1}, \gamma_{2}\right) \in N$ and $\gamma_{1} \gamma_{2}=\gamma$.

Let $M^{1}=\bar{M}$ and for $k>1$ let

$$
M^{k}=M^{k-1} \cup\left(M^{k-1} \cdot \bar{M}\right),
$$

that is, $(x, \gamma) \in M^{k}$, if and only if there exist $l \leq k$ and $\gamma_{1}, \ldots, \gamma_{l} \in \Gamma$ such that $\gamma_{1} \cdots \gamma_{l}=\gamma$ and

$$
\left(x \gamma_{1} \cdots \gamma_{i}, \gamma_{i+1}\right) \in M \quad \text { or } \quad\left(x \gamma_{1} \cdots \gamma_{i} \gamma_{i+1}, \gamma_{i+1}^{-1}\right) \in M \quad(i<l) .
$$

A graphing $M$ is an L-graphing if

$$
\bigcup_{k} M^{k}=X \times \Gamma
$$

The groupoid cost of $(\Gamma, X)$ is defined as

$$
\operatorname{gcost}(\Gamma, X)=\inf e(M)
$$

where the infimum is taken over all $L$-graphings $M$.

A graphing $M$ is finitely supported if $M_{\gamma}$ is empty for all but finitely many $\gamma \in \Gamma$. The distance of two graphings $M, N$ is defined as

$$
d(M, N)=e(M \triangle N)=\sum_{\gamma \in \Gamma} \mu\left(M_{\gamma} \Delta N_{\gamma}\right)
$$

where $A \triangle B$ denotes the symmetric difference of $A$ and $B$.

Let us fix a base $O$ of the topology of $X$. We call a subset of $X$ cylindric with respect to $O$ if it is a finite union of elements of $O$. A graphing $M$ is cylindric if for all $\gamma \in \Gamma$ the set $M_{\gamma}$ is cylindric.

The following lemma says that under some assumptions on $X$ and $\Gamma$, every $L$-graphing can be approximated by open finitely supported cylindric $L$-graphings. Note that we do not assume that $X$ is a standard Borel space: it can also be a finite set.

Lemma 5. Assume that $X$ is compact, $\Gamma$ is finitely generated and acts by homeomorphisms on $X$. Let $M$ be an L-graphing of finite measure and let $O$ be a base of the topology on $X$. Then for all $\varepsilon>0$ there exists an open finitely supported L-graphing $N$ that is cylindric with respect to $O$ and such that $d(M, N)<\varepsilon$.

Proof. Fix a generating set $g_{1}, \ldots, g_{d}$ of $\Gamma$. Let $B$ denote the graphing defined by

$$
B_{\gamma}= \begin{cases}X, & \gamma=g_{i} \text { for some } i \leq d, \\ \varnothing, & \text { otherwise. }\end{cases}
$$

Clearly, $B$ is an $L$-graphing of measure $d$. List the elements of $\Gamma$ as $\gamma_{1}, \gamma_{2}, \ldots$ and the elements of the base $O$ as $O_{1}, O_{2}, \ldots$. 
Let $K \supseteq M$ be an open graphing satisfying $e(K \backslash M)<\varepsilon / 3$. For $n \geq 0$ let the finitely supported graphing $K(n)$ be defined by

$$
K(n)_{\gamma}= \begin{cases}K_{\gamma}, & \gamma=\gamma_{i} \text { for some } i \leq n \\ \emptyset, & \text { otherwise. }\end{cases}
$$

Since $K$ has finite edge-measure, it follows that

$$
\lim _{n \rightarrow \infty} d(K(n), K)=0 .
$$

For $d \geq 2$, we have $K^{d}=\bigcup_{n} K(n)^{d}$, which yields

$$
\bigcup_{n} K(n)^{n}=\bigcup_{n} K^{n}=X \times \Gamma \supseteq B .
$$

It is easy to see that $K(n)^{n}$ is open and $K(n)^{n} \subseteq K(n+1)^{n+1}(n \geq 2)$. Since $B$ is compact, there exists $k$ such that, setting $L=K(k)$, we have $d(L, K)<\varepsilon / 3$ and $L^{k} \supseteq B$. This implies that

$$
\bigcup_{n} K^{n} \supseteq \bigcup_{n} B^{n}=X \times \Gamma,
$$

that is, $L$ is an $L$-graphing.

For $n \geq 1$ let the graphing $L(n)$ be defined by

$$
L(n)_{\gamma}=\bigcup_{\substack{1 \leq i \leq n \\ O_{i} \subseteq L_{\gamma}}} O_{i}
$$

Then $L(n)$ is a finitely supported cylindric graphing $(n \geq 1)$ and $\bigcup_{n} L(n)=L$. So, using the same argument as above we have

$$
\bigcup_{n} L(n)^{n}=\bigcup_{n} L^{n}=X \times \Gamma \supseteq B
$$

and using the compactness of $B$ again, there exists $k$ such that, setting $N=L(k)$, we have both $N^{k} \supseteq B$ and $d(N, L)<\varepsilon / 3$. So $N$ is an $L$-graphing of distance at most $\varepsilon$ from $M$ and the lemma is proved.

Now we will analyze the connection between Borel subgraphs and graphings. Every graphing $M$ defines a Borel subgraph $\Phi(M)$ of $E$ as follows:

$$
\Phi(M)=\{(x, x \cdot \gamma) \mid(x, \gamma) \in M\} .
$$

As we will see, the map $\Phi$ is surjective. Note that it is bijective if and only if the action of $\Gamma$ on $X$ is free.

Lemma 6. We have

$$
\operatorname{gcost}(\Gamma, X) \geq \operatorname{cost}(\Gamma, X) .
$$

If $\Gamma$ acts essentially freely on $X$, then we have equality. 
Proof. Let $M$ be a graphing. It is easy to see that

$$
\Phi\left(M^{k}\right)=\Phi(M)^{k} \quad(k \geq 2) .
$$

This implies that if $M$ is an $L$-graphing then $\Phi(M)$ is a Borel subgraph of $E$ that spans $E$.

Now for $x \in X$,

$$
\begin{aligned}
\operatorname{deg}_{S} x & =\mid\left\{y \in X \mid \exists \gamma \in \Gamma \text { with } x \in M_{\gamma} \text { and } y=x \cdot \gamma\right\} \mid \\
& \leq\left|\left\{\gamma \in \Gamma \mid x \in M_{\gamma}\right\}\right|=\operatorname{deg}_{M}(x)
\end{aligned}
$$

which implies $e(\Phi(M)) \leq e(M)$. It follows that $\operatorname{gcost}(\Gamma, X) \geq \operatorname{cost}(\Gamma, X)$.

Assume that $\Gamma$ acts essentially freely on $X$. Let us list the elements of $\Gamma$ as $\gamma_{1}, \gamma_{2}, \ldots$ Let $S$ be a Borel subgraph of $E$ spanning $E$. For each $(x, y) \in S$ let us define $f(x, y)$ to be the first element of $\Gamma$ such that

$$
y=x \cdot f(x, y) .
$$

Let us define

$$
M=\{(x, \gamma) \in X \times \Gamma \mid f(x, x \cdot \gamma)=\gamma\} .
$$

Then $M$ is a graphing that satisfies $\Phi(M)=S$. Also, for almost all $x \in X$ there is equality in (1), which yields

$$
e(M)=e(S) .
$$

However, the graphing $M$ may not be an $L$-graphing. Let

$$
N=\bigcup_{n} M^{n}
$$

Then

$$
\Phi(N)=\bigcup_{n} \Phi\left(M^{n}\right)=\bigcup_{n} \Phi(M)^{n}=\bigcup_{n} S^{n}=E .
$$

Let $(x, \gamma) \in(X \times \Gamma) \backslash N$. Then $(x, x \cdot \gamma) \in E=\Phi(N)$ so for some $\delta \in \Gamma$ such that $(x, \delta) \in N$ we have $x \cdot \gamma=x \cdot \delta$, implying $\gamma \delta^{-1} \in \operatorname{Stab}_{\Gamma}(x)$. Moreover $\delta \neq \gamma$ since $(x, \gamma) \notin N$. Thus $x \in X$ has nontrivial stabilizer in $\Gamma$. Since the action of $\Gamma$ is essentially free, we obtain $e((X \times \Gamma) \backslash N)=0$. But then

$$
M^{\prime}=M \cup((X \times \Gamma) \backslash N)
$$

is an $L$-graphing of measure $e\left(M^{\prime}\right)=e(S)$. This implies

$$
\operatorname{gcost}(\Gamma, X) \leq \operatorname{cost}(\Gamma, X),
$$

so equality holds as claimed. 


\section{Boundary action and rank gradient}

In this section we first introduce coset trees and boundary representations. Up to the authors' knowledge, the first reference for studying ergodic properties of boundary actions is the fairly recent work of Grigorchuk, Nekrashevich and Suschanskii [12]. Then we express the groupoid cost of a boundary representation in terms of the rank gradient of the chain. This allows us to prove Theorem 1.

Let $\left(\Gamma_{n}\right)$ be a chain in $\Gamma$. Then the coset tree $T=T\left(\Gamma,\left(\Gamma_{n}\right)\right)$ of $\Gamma$ with respect to $\left(\Gamma_{n}\right)$ is defined as follows. The vertex set of $T$ equals

$$
T=\left\{\Gamma_{n} g \mid n \geq 0, g \in \Gamma\right\}
$$

and the edge set is defined by inclusion, that is,

$$
\left(\Gamma_{n} g, \Gamma_{m} h\right) \text { is an edge in } T \quad \text { if } \quad m=n+1 \text { and } \Gamma_{n} g \supseteq \Gamma_{m} h .
$$

Then $T$ is a tree rooted at $\Gamma$ and every vertex of level $n$ has the same number of children, equal to the index $\left|\Gamma_{n}: \Gamma_{n+1}\right|$. The right actions of $\Gamma$ on the coset spaces $\Gamma / \Gamma_{n}$ respect the tree structure and so $\Gamma$ acts on $T$ by automorphisms. This action is called the tree representation of $\Gamma$ with respect to $\left(\Gamma_{n}\right)$.

The boundary $\partial T$ of $T$ is defined as the set of infinite rays starting from the root. The boundary is naturally endowed with the product topology and product measure coming from the tree. More precisely, for $t=\Gamma_{n} g \in T$ let us define $\operatorname{Sh}(t) \subseteq \partial T$, the shadow of $t$, as

$$
\operatorname{Sh}(t)=\{x \in \partial T \mid t \in x\}
$$

the set of rays going through $t$. Set the base of topology on $\partial T$ to be the set of shadows and set the measure of a shadow to be

$$
\mu(\operatorname{Sh}(t))=1 /\left|\Gamma: \Gamma_{n}\right| .
$$

This turns $\partial T$ into a totally disconnected compact space with a Borel probability measure $\mu$. The group $\Gamma$ acts on $\partial T$ by measure-preserving homeomorphisms; we call this action the boundary representation of $\Gamma$ with respect to $\left(\Gamma_{n}\right)$. The following lemma already appears in [12]; we include the proof for completeness.

Lemma 7. The action of $\Gamma$ on $\partial T$ is ergodic and minimal (that is, every orbit is dense).

Proof. Let $A \subseteq \partial T$ be a measurable $\Gamma$-invariant subset such that $\mu(A)>0$. Then using the Lebesgue density theorem, for all $\varepsilon>0$ there exists $t \in T$ of level $n$ with

$$
\mu(\operatorname{Sh}(t) \cap A) \geq(1-\varepsilon) \mu(\operatorname{Sh}(t)) .
$$

Since $\Gamma$ acts transitively on the $n$-th level of $T$, invariance implies the same inequality for all $u \in T$ of level $n$. Adding up, we get

$$
\mu(A)=\sum_{u \in T \text { of level } n} \mu(\operatorname{Sh}(u) \cap A) \geq 1-\varepsilon,
$$

which implies $\mu(A)=1$. 
Now let $x \in \partial T$ and let $t \in T$. Let $t^{\prime} \in T$ be the vertex of the same level as $t$ contained in $x$ and let $g \in \Gamma$ with $t^{\prime} g=t$. Then $x g \in \operatorname{Sh}(t)$. We have proved that the orbit of $x$ is dense in $\partial T$.

There are various levels of faithfulness of a boundary representation. Let

$$
\partial T_{\text {free }}=\left\{x \in \partial T \mid \operatorname{Stab}_{\Gamma}(x)=1\right\} .
$$

We say that the action is essentially free (or that the chain is Farber) if $\mu\left(\partial T \backslash \partial T_{\text {free }}\right)=0$. The action is topologically free if $\partial T \backslash \partial T_{\text {free }}$ is meagre, i.e., a countable union of nowhere dense closed sets. The action is free if $\partial T_{\text {free }}=\partial T$. Note that the Farber condition has been introduced by Farber in [9] in another equivalent formulation (see also [6] for a relevant result).

It is easy to see that the following implications hold for the action of $\Gamma$ on $\partial T$ :

$\left(\Gamma_{n}\right)$ is normal and $\bigcap \Gamma_{n}=1 \Rightarrow$ free $\Rightarrow$ essentially free

$$
\Rightarrow \text { topologically free } \Leftrightarrow X_{\text {free }} \neq \emptyset \Rightarrow \text { faithful, }
$$

For all but the third arrow it is easy to find examples showing that the reverse implications do not hold. We shall discuss these classes more in Section 4.

Note that a deep result of Stuck and Zimmer [30] tells us that every faithful ergodic measure-preserving action of a higher rank semisimple real lattice on a probability space is essentially free. In particular, every faithful boundary representation of such a lattice is essentially free.

Let $\Gamma$ be a group and let $X$ be a set. A directed $\Gamma$-labelled graph is a triple $(V, E, f)$ where $(V, E)$ is a directed graph with vertex set $V$ and edge list $E$ and $f$ is a function from $E$ to $\Gamma$. Note that we allow multiple edges and we make no restriction on the labelling $f$. For a directed $\Gamma$-labelled graph

$$
G=\left(V, E=\left(e_{1}, \ldots, e_{n}\right), f\right)
$$

let $U(G)=(V, U(E))$ denote the undirected graph with vertex set $V$ and edge list

$$
U(E)=\left(\overline{e_{1}}, \ldots, \overline{e_{n}}\right)
$$

where $\bar{e}$ denotes the unordered pair obtained from the ordered pair $e$.

Let $G$ be a directed $\Gamma$-labelled graph and let $v \in V$. Then we can define a natural map

$$
\Phi_{v}: \pi_{1}(U(G), v) \rightarrow \Gamma
$$

from the fundamental group of $U(G)$ based at $v$ to $\Gamma$ as follows. For a loop $l=\left(e_{1}, \ldots, e_{k}\right)$ in $U(G)$ starting at $v$ let

$$
\Phi_{v}(l)=\prod_{i=0}^{n-1} f^{ \pm 1}\left(e_{i}\right)
$$

where the sign depends on whether we travel along $e_{i}$ preserving its original orientation in $G$ or not. The following lemma is straightforward. 
Lemma 8. The map $\Phi_{v}$ is a group homomorphism.

Now we express the rank gradient of a chain in terms of the groupoid cost of the action on the boundary of the coset tree. Note that we do not make any assumptions on the boundary representation. In fact, we do not even assume that the chain is infinite!

Theorem 9. Let $\left(\Gamma_{n}\right)$ be any chain in $\Gamma$. Then

$$
\operatorname{RG}\left(\Gamma,\left(\Gamma_{n}\right)\right)=\operatorname{gcost}(E)-1
$$

where $E=E\left(\partial T\left(\Gamma,\left(\Gamma_{n}\right)\right)\right)$ denotes the orbit relation on $\partial T\left(\Gamma,\left(\Gamma_{n}\right)\right)$ defined by the action of $\Gamma$.

Proof. Let $s=\operatorname{RG}\left(\Gamma,\left(\Gamma_{n}\right)\right)$ and let $c=\operatorname{gcost}(E)$.

First we show $s+1 \geq c$. Let $\varepsilon>0$. Then there exists $n$ such that

$$
\frac{d\left(\Gamma_{n}\right)-1}{\left|\Gamma: \Gamma_{n}\right|}<s+\varepsilon
$$

that is, $\Gamma_{n}$ can be generated by at most

$$
d=\left\lfloor(s+\varepsilon)\left|\Gamma: \Gamma_{n}\right|\right\rfloor+1
$$

elements, where $\lfloor x\rfloor$ is the floor of $x$. Let $h_{1}, \ldots, h_{d}$ be such a generating set and let

$$
\gamma_{1}, \ldots, \gamma\left|\Gamma: \Gamma_{n}\right|
$$

be a coset representative system for $\Gamma_{n}$ in $\Gamma$. We can assume that $\gamma_{1}=1$. Let

$$
Y=\left\{h_{i} \mid i \leq d\right\} \cup\left\{h_{i}^{-1} \mid i \leq d\right\} \cup\{1\} .
$$

Let us define a graphing $M$ as follows:

$$
M_{\gamma}= \begin{cases}\operatorname{Sh}\left(\Gamma_{n}\right) & \text { if } \gamma=h_{i}(i \geq 1) \text { or } \gamma=\gamma_{i}(i>1), \\ \varnothing & \text { otherwise. }\end{cases}
$$

We claim that $M$ is an $L$-graphing. First, we have

$$
\bar{M}_{\gamma} \supseteq \begin{cases}\operatorname{Sh}\left(\Gamma_{n}\right) & \text { if } \gamma \in Y, \\ \operatorname{Sh}\left(\Gamma_{n}\right) & \text { if } \gamma=\gamma_{i}\left(i \leq\left|\Gamma: \Gamma_{n}\right|\right), \\ \operatorname{Sh}\left(\Gamma_{n} \gamma_{i}\right) & \text { if } \gamma=\gamma_{i}^{-1}\left(i \leq\left|\Gamma: \Gamma_{n}\right|\right) .\end{cases}
$$

Let $(x, \gamma) \in \partial T \times \Gamma$. Then there exists $a, b \leq\left|\Gamma: \Gamma_{n}\right|$ such that $x \in \operatorname{Sh}\left(\Gamma_{n} \gamma_{a}\right)$ and $\gamma_{a} \gamma \gamma_{b}^{-1} \in \Gamma_{n}$. This implies that there are elements $y_{1}, \ldots, y_{k} \in Y$ such that $\gamma_{a} \gamma \gamma_{b}^{-1}=$ $y_{1} \cdots y_{k}$. Using that $Y \subseteq \Gamma_{n}$ we get

$$
(x, \gamma)=\left(x, \gamma_{a}^{-1} y_{1} \cdots y_{k} \gamma_{b}\right) \in \bar{M}^{k+2} .
$$

Now the edge-measure of $M$ equals

$$
e(M)=\frac{1}{\left|\Gamma: \Gamma_{n}\right|}\left(d+\left|\Gamma: \Gamma_{n}\right|-1\right) \leq 1+s+\varepsilon,
$$

which implies $c \leq 1+s$ as claimed. 
Now we show that $s \leq c-1$. Let $\varepsilon>0$. Then there exists an $L$-graphing $M$ of $E$ of edge-measure at most $c+\varepsilon / 2$. By Lemma 5 (setting the set of shadows as base of the topology) there exists a finitely supported cylindric $L$-graphing $N$ of $E$ with $d(M, N)<$ $\varepsilon / 2$. This implies that $e(N)<c+\varepsilon$.

Let $n$ be a natural number such that for every $\gamma \in \Gamma$ the set $N_{\gamma}$ is a union of shadows of some cosets of $\Gamma_{n}$. Let

$$
V=\left\{\Gamma_{n} \gamma \mid \gamma \in \Gamma\right\} \quad \text { and } \quad v=\Gamma_{n}
$$

Let us define the undirected $\Gamma$-labelled graph $G=(V, E, f)$ as follows. For each $\gamma \in \Gamma$ and $w \in V$ where $\operatorname{Sh}(w) \subseteq N_{\gamma}$ let us add the edge $(w, w \gamma)$ to the list $E$ with label $f(w, w \gamma)=\gamma$.

Let us consider the map $\Phi_{v}: \pi_{1}(U(G), v) \rightarrow \Gamma$. We claim that the image of $\Phi_{v}$ is

$$
\Phi_{v}\left(\pi_{1}(U(G), v)\right)=\Gamma_{n} .
$$

First, let $l=\left(e_{1}, \ldots, e_{k}\right)$ be a loop in $U(G)$ starting at $v$. Then $v \Phi_{v}(l)=v$ so $\Phi_{v}(l)$ $\in \Gamma_{n}$.

Second, let $h \in \Gamma_{n}$. Let $x \in \operatorname{Sh}(v)$ be an arbitrary element. Then since $N$ is an $L$ graphing, we have $(x, h) \in N^{k}$ for some $k$. Thus there exist $\gamma_{1}, \ldots, \gamma_{k} \in \Gamma$ such that $\gamma_{1} \cdots \gamma_{k}=h$ and

$$
\left(x \gamma_{1} \cdots \gamma_{i}, \gamma_{i+1}\right) \in N \quad \text { or } \quad\left(x \gamma_{1} \cdots \gamma_{i} \gamma_{i+1}, \gamma_{i+1}^{-1}\right) \in N \quad(i<k)
$$

Let $x_{i}=x \gamma_{1} \cdots \gamma_{i}(0 \leq i \leq k)$. Then $v h=v$ so $x_{0}=v=x_{k}$. Also, for all $0 \leq i<k$ there is an edge in $G$ either from $x_{i}$ to $x_{i+1}$ labelled by $\gamma_{i+1}$ or from $x_{i+1}$ to $x_{i}$ labelled by $\gamma_{i+1}^{-1}$. Thus these edges form a loop $l$ in $U(G)$ with $\Phi_{v}(l)=h$. The claim follows.

The number of vertices of $G$ equals $\left|\Gamma: \Gamma_{n}\right|$ while the number of edges of $G$ equals $e(N)\left|\Gamma: \Gamma_{n}\right|$. Hence, the same holds for $U(G)$. Using Lemma 8 and the formula for the rank of the fundamental group of a graph we get

$$
d\left(\Gamma_{n}\right) \leq d\left(\pi_{1}(U(G), v)\right)=e(N)\left|\Gamma: \Gamma_{n}\right|-\left|\Gamma: \Gamma_{n}\right|+1,
$$

which yields

$$
\frac{d\left(\Gamma_{n}\right)-1}{\left|\Gamma: \Gamma_{n}\right|} \leq e(N)-1<c-1+\varepsilon .
$$

This shows that $s \leq c-1$.

Now Theorem 1 follows immediately:

Proof of Theorem 1. Using Theorem 9 and Lemma 6 we get

$$
\operatorname{RG}\left(\Gamma,\left(\Gamma_{n}\right)\right)=\operatorname{gcost}(E)-1=\operatorname{cost}(E)-1 .
$$

\section{Applications and examples}

In this section we introduce the absolute rank gradient of a group and compute it for some important classes of groups. We will later use these results in Section 5. Then we discuss what happens if we relax the Farber condition. 
Let us define the absolute rank gradient of $\Gamma$ as

$$
\mathrm{RG}(\Gamma)=\inf _{H} \frac{d(H)-1}{|\Gamma: H|}
$$

where $H$ runs through all subgroups of $\Gamma$ of finite index.

Let $H, K \leq \Gamma$ be subgroups of finite index with $H \leq K$. Using the Nielsen-Schreier theorem on $H$ and $K$, we get $d(H)-1 \leq|K: H|(d(K)-1)$, which yields

$$
\frac{d(H)-1}{|\Gamma: H|} \leq \frac{d(K)-1}{|\Gamma: K|} .
$$

So for any chain $\left(\Gamma_{n}\right)$ in $\Gamma$, the sequence $\left(d\left(\Gamma_{n}\right)-1\right) /\left|\Gamma: \Gamma_{n}\right|$ is nonincreasing and the definition of $\operatorname{RG}\left(\Gamma,\left(\Gamma_{n}\right)\right)$ makes sense. In fact, as the authors show in [2], if the sequence stabilizes then $\Gamma$ is virtually free.

We shall make use of the following easy lemma.

Lemma 10. Let $\Gamma$ be a finitely generated, residually finite group and let $H \leq \Gamma$ be a subgroup of finite index. Then there exists a chain $\left(\Gamma_{n}\right)$ in $\Gamma$ such that $\Gamma_{1}=H$ and the boundary representation of $\Gamma$ with respect to $\left(\Gamma_{n}\right)$ is free.

Proof. Let $K$ be the core of $H$, that is, $K=\bigcap_{g \in \Gamma} H^{g}$. Then $K$ is a normal subgroup of $\Gamma$ of finite index. For $n \geq 1$ let

$$
\Delta_{n}=\bigcap_{L \leq \Gamma \text { of index } n} L .
$$

Since $\Gamma$ has only finitely many subgroups of a given index, each $\Delta_{n}$ is a normal subgroup of finite index in $\Gamma$. Also, since $\Gamma$ is residually finite, $\bigcap_{n} \Delta_{n}=1$. Now let $\Gamma_{0}=\Gamma$, let $\Gamma_{1}=H$ and for $n \geq 2$ let $\Gamma_{n}=K \bigcap \Delta_{n}$. Then $\bigcap_{n} \Gamma_{n}=1$ and the chain $\left(\Gamma_{n}\right)$ consists of normal subgroups of $\Gamma$ (except for $n=1$ ). Let $g \in \Gamma$ with $g \neq 1$. Then there exists $n>1$ such that $g \notin \Gamma_{n}$. This implies that $g$ acts fixed point freely on $\Gamma / \Gamma_{n}$ and thus also on the boundary $\partial T\left(\Gamma,\left(\Gamma_{n}\right)\right)$. In other words, the action of $\Gamma$ on $\partial T$ is free.

Theorem 1 now gives us the following on the absolute rank gradient.

Corollary 11. Let $\Gamma$ be a finitely generated, residually finite group. Then

$$
\operatorname{RG}(\Gamma)=\operatorname{cost}(\Gamma, \widehat{\Gamma})-1
$$

where $\widehat{\Gamma}$ denotes the profinite completion of $\Gamma$.

Proof. Let $\Delta_{n}$ be as in the proof of Lemma 10. Then for all $H \leq \Gamma$ of index $n$, we have $\Delta_{n} \leq H$. Hence $\widehat{\Gamma}_{\left(\Delta_{n}\right)}$, the profinite completion of $\Gamma$ with respect to $\left(\Delta_{n}\right)$, equals $\widehat{\Gamma}$ and by Theorem 1 we get

$$
\operatorname{RG}(\Gamma)=\operatorname{RG}\left(\Gamma, \Delta_{n}\right)=\operatorname{cost}\left(\Gamma, \widehat{\Gamma}_{\left(\Delta_{n}\right)}\right)-1=\operatorname{cost}(\Gamma, \widehat{\Gamma})-1 .
$$

Proof of Corollary 3. Let $\Gamma$ be a finitely generated residually finite group with an infinite amenable normal subgroup. Let $\left(\Gamma_{n}\right)$ be a Farber chain in $\Gamma$-such a chain exists by 
Lemma 10. Let $E=E\left(\partial T\left(\Gamma,\left(\Gamma_{n}\right)\right)\right)$. Then by [11], $\Gamma$ has fixed price 1 and so, using Theorem 1, we have

$$
\mathrm{RG}(\Gamma)=\operatorname{RG}\left(\Gamma,\left(\Gamma_{n}\right)\right)=\operatorname{cost}(E)-1=0 .
$$

Note that in a joint paper [2] with A. Jaikin-Zapirain we present an interesting alternative combinatorial proof of the corollary in the case when $\Gamma$ itself is infinite amenable. However, we have no proof for the general case that does not use analysis.

Ascending HNN extensions. Let $A$ be a finitely generated group and $f: A \rightarrow A$ an injective homomorphism. Then the ascending HNN extension with base $A$ is the group $\Gamma$ with presentation

$$
\left\langle A, t \mid a^{t}=f(a) \forall a \in A\right\rangle
$$

For example every free-by-cyclic group has such a presentation.

For each $n \in \mathbb{N}$ let $\Gamma_{n}=\left\langle A, t^{n}\right\rangle \leq \Gamma$. It is easy to see that $\Gamma_{n}$ is a normal subgroup of index $n$ in $\Gamma$. As $d\left(\Gamma_{n}\right) \leq d(A)+1$ we get the following result, first observed by Lackenby in [17].

Proposition 12. If the group $\Gamma$ is a residually finite ascending HNN extension, then $\mathrm{RG}(\Gamma)=0$.

For instance, free-by-cyclic groups are residually finite and so they have rank gradient 0 . In fact, more generally we have the following.

Proposition 13. Let $\Gamma$ be finitely generated residually finite group which has a finitely generated normal subgroup $N$ such that $\Gamma / N$ has subgroups of arbitrarily large index (i.e. the profinite completion $\widehat{\Gamma / N}$ of $\Gamma / N$ is infinite). Then $\operatorname{RG}(\Gamma)=0$.

Proof. Using the assumptions one can find a sequence of subgroups $H_{i}<\Gamma$ such that both

$$
a_{i}=\left[\Gamma: N H_{i}\right] \quad \text { and } \quad b_{i}=\left[N: N \cap H_{i}\right]
$$

tend to infinity as $i \rightarrow \infty$. Now $\left[\Gamma: H_{i}\right]=a_{i} b_{i}, d\left(N \cap H_{i}\right) \leq b_{i} d(N)$ and $d\left(N H_{i} / N\right) \leq$ $a_{i} d(\Gamma)$. Hence from $d\left(H_{i}\right) \leq d\left(N \cap H_{i}\right)+d\left(N H_{i} / N\right)$ we obtain

$$
\frac{d\left(H_{i}\right)-1}{\left[\Gamma: H_{i}\right]}<\frac{d(\Gamma)}{b_{i}}+\frac{d(N)}{a_{i}},
$$

which tends to 0 as $i \rightarrow \infty$. This implies $\operatorname{RG}(\Gamma)=0$.

The fact that the groups in Proposition 13 have a measurable action with cost 1 has been proved by Gaboriau [11], even without assuming residual finiteness of $\Gamma$ and $|\widehat{\Gamma / N}|=\infty$, but that does not give anything on the rank gradient. We note that the condition that $\widehat{\Gamma / N}$ is infinite seems quite mild.

Now we discuss rank gradient of chains that are not Farber. As we shall see, the situation can be quite different.

Our first example is the so-called lamplighter group, the wreath product

$$
\Gamma=\mathbb{Z} / 2 \mathbb{Z} 2 \mathbb{Z} .
$$


This group is metabelian, in particular, it is amenable, so Corollary 3 implies that the rank gradient of any Farber chain is 0. However, consider the canonical surjections

$$
\phi_{n}: \mathbb{Z} / 2 \mathbb{Z} \imath \mathbb{Z} \rightarrow \mathbb{Z} / 2 \mathbb{Z} \imath \mathbb{Z} / 2^{n} \mathbb{Z} \text { and } \pi_{n}: \mathbb{Z} / 2 \mathbb{Z} \imath \mathbb{Z} / 2^{n} \mathbb{Z} \rightarrow \mathbb{Z} / 2^{n} \mathbb{Z}
$$

and let $\Gamma_{n}=\operatorname{Ker}\left(\phi_{n} \pi_{n}\right)$. Then $\left(\Gamma_{n}\right)$ is a normal chain, $\Gamma / \Gamma_{n} \approx \mathbb{Z} / 2^{n} \mathbb{Z}$ and $\phi_{n}\left(\Gamma_{n}\right) \approx$ $(\mathbb{Z} / 2 \mathbb{Z})^{2^{n}}$, which implies $d\left(\Gamma_{n}\right) \geq 2^{n}$. This yields

$$
\operatorname{RG}\left(\Gamma,\left(\Gamma_{n}\right)\right) \geq 1
$$

In the above example the chain is normal but with nontrivial intersection, and so the boundary representation is not faithful. We do not know whether there exists an amenable group $\Gamma$ and a chain $\left(\Gamma_{n}\right)$ such that the boundary representation is faithful, but the chain has positive rank gradient.

Our last example is a slight variation of one discussed by Bergeron and Gaboriau in [6]. The difference is that they estimate the first Betti number where we estimate the rank.

Proposition 14. There exist a virtually free group $\Gamma$ and an interval $[x, y) \subset \mathbb{R}_{+}$such that for every $\alpha \in[x, y)$ there exists a subnormal chains of subgroups $\Gamma=H_{0}>H_{1}>$ $H_{2}>\cdots$ with trivial intersection such that $\operatorname{RG}\left(\Gamma,\left(H_{i}\right)\right)=\alpha$.

Moreover in this situtation we can have the strict inequalities

$$
\operatorname{RG}\left(\Gamma,\left(H_{i}\right)\right)>\lim _{i \rightarrow \infty} \frac{b_{1, p}\left(H_{i}\right)}{\left|\Gamma: H_{i}\right|}>\lim _{i \rightarrow \infty} \frac{b_{1}\left(H_{i}\right)}{\left|\Gamma: H_{i}\right|},
$$

where for a prime $p$ the integer $b_{1, p}(H)=\operatorname{dim}_{\mathbb{F}_{p}} H /[H, H] H^{p}$ is the p-homology of $H$.

In particular, these chains are not Farber.

Proof of Proposition 14. Let $\Gamma=A * \mathbb{Z}$, where $A$ is a finite group. Viewing $\Gamma$ as a trivial HNN extension we see that $\Gamma$ has a right transitive action on both the vertices and the edges of a regular $2|A|$-valent tree $T$ (so that the quotient $I_{0}$ is a single vertex with a looped edge). We can direct the edges of $T$ so that $\Gamma$ acts preserving this orientation. There are exactly $a=|A|$ in-edges and out-edges from every vertex of $T$. The action of $\Gamma$ is regular on the edges of $T$. We fix an edge $e_{0}$ of $T$ and a vertex $v_{0}$ at one end of $e_{0}$ so that the stabilizer of $v_{0}$ is $A$.

Suppose that $H$ is a subgroup of $\Gamma$ of index $n$. Then the quotient $I=T / H$ is a finite graph with a covering map $p: T \rightarrow I=T / H$ and $H$ can be recovered from $p$ as $\left\{g \in \Gamma \mid p\left(e_{0}\right)=p\left(e_{0} g\right)\right\}$.

The vertices of $I$ are in 1-1 correspondence with the double cosets $A \backslash \Gamma / H$. Given a vertex $v \in I$ define $S_{v}=A^{g} \cap H$. where $g$ is a fixed representative of $A \backslash \Gamma / H$ such that $p\left(v_{0} g\right)=v$. There are exactly $n$ edges in $I$ and we have $n=\sum_{v \in I}\left[A: S_{v}\right]$.

The Bass-Serre theory determines the structure of $H$ as follows:

$$
H=F *\left(\underset{v \in I}{*} S_{v}\right)
$$


where the free group $F$ is the fundamental group of $I$. The group $F$ has rank $n-p+1$, where $p$ is the number of vertices in $I$.

Assuming that every vertex of $I$ has out-valency 1 or $a$, let $X$ be the set of vertices of $I$ with out-valency 1 and let $Y=I \backslash X$ be those with out-valency $a=|A|$. Let $|X|=\mu p$ for some $\mu \in[0,1]$. Then we have

$$
n=p(\mu+(1-\mu)|A|), \quad \text { hence } \quad p=\frac{n}{\mu+(1-\mu)|A|} .
$$

We find that exactly $\mu p$ of the vertex stabilizers $S_{v}$ are equal to $A$ and the rest are trivial. From the Grushko-Neumann theorem (see [13] and [21]) it now follows that the rank of $H$ is

$$
d(H)=n-p+\mu p d(A)+1=n\left(1+\frac{\mu d(A)-1}{\mu+(1-\mu)|A|}\right)+1
$$

while $\beta_{1}(H)=n-p+1$.

Now suppose that we have a sequence of finite oriented graphs with covering maps

$$
I_{0} \leftarrow I_{1} \leftarrow I_{2} \leftarrow \cdots \leftarrow T
$$

with the following two properties:

1. Each vertex of each $I_{j}$ has valency 2 or $2 a$. The proportions $\mu_{j}=\left|X_{j}\right| /\left|I_{j}\right|$ of the vertices of $I_{j}$ with out- and in-valency 1 form a decreasing sequence which tends to some prescribed limit $\mu=\mu_{\infty} \in[0,1)$.

2. Given any integer $k$ there exists some $m \in \mathbb{N}$ such that the covering map $T \rightarrow I_{m}$ is injective on $B_{k}$, where $B_{k}$ is the ball of radius $k$ in the $2 a$-regular oriented tree $T$ centred at the vertex $v_{0}$.

Then the groups $H_{j}$ which correspond to the graphs $I_{j}$ form a chain in $\Gamma$ with trivial intersection and such that

$$
\lim _{i \rightarrow \infty} \frac{d\left(H_{i}\right)-1}{\left|\Gamma: H_{i}\right|}=1+\frac{\mu d(A)-1}{\mu+(1-\mu)|A|}
$$

The first part of Proposition 14 now follows by setting $x=1-1 /|A|\left(\right.$ when $\left.\mu_{\infty}=0\right)$ and $y=d(A)$ (when $\mu_{\infty} \rightarrow 1$ ).

The existence of the graphs $I_{j}$ with properties 1 and 2 above is essentially proved by Bergeron and Gaboriau in [6, Section 4] in the case of a free product of two residually finite groups.

On the other hand, we have

$$
\lim _{i \rightarrow \infty} \frac{\beta_{1}\left(H_{i}\right)}{\left|\Gamma: H_{i}\right|}=1-\frac{1}{\mu+(1-\mu)|A|}
$$

and similarly

$$
b_{1, p}(H)=n\left(1+\frac{\mu b_{1, p}(A)-1}{\mu+(1-\mu)|A|}\right)+1 .
$$


Therefore

$$
\lim _{i \rightarrow \infty} \frac{\beta_{1, p}\left(H_{i}\right)}{\left|\Gamma: H_{i}\right|}=1+\frac{\mu \beta_{1, p}(A)-1}{\mu+(1-\mu)|A|} .
$$

So, if we choose the finite group $A$ so that $d(A)>b_{1, p}(A)>0$ and choose the chain $\left(H_{i}\right)$ with limiting ratio $\mu_{\infty}>0$, this gives

$$
\operatorname{RG}\left(\Gamma,\left(H_{i}\right)\right)>\lim _{i \rightarrow \infty} \frac{b_{1, p}\left(H_{i}\right)}{\left|\Gamma: H_{i}\right|}>\lim _{i \rightarrow \infty} \frac{b_{1}\left(H_{i}\right)}{\left|\Gamma: H_{i}\right|}
$$

as promised.

\section{Lattices and 3-manifolds}

In this section we further discuss the rank gradient, with a special emphasis on lattices in Lie groups. We also define the Heegaard genus and derive the contradiction between the rank vs. Heegaard genus conjecture and fixed price.

The following lemma follows immediately from [11, Theorem 3] saying that if $H \leq \Gamma$ has finite index, then

$$
\operatorname{cost}(H)-1=|\Gamma: H|(\operatorname{cost}(\Gamma)-1)
$$

where $\operatorname{cost}(\Gamma)$ is the infimum of costs of all measurable essentially free actions of $\Gamma$.

Lemma 15. An affirmative answer to the fixed price problem implies an affirmative answer to the multiplicativity of cost-minus-1 problem.

Proof. Let $\Gamma$ be a finitely generated group acting on $(X, \mu)$ by measure-preserving maps and let $H$ be a subgroup of $\Gamma$ of finite index. Then by our assumption we have

$$
\operatorname{cost}(H, X)-1=\operatorname{cost}(H)-1=|\Gamma: H|(\operatorname{cost}(\Gamma)-1)=|\Gamma: H|(\operatorname{cost}(\Gamma, X)-1) .
$$

Now we proceed to the independence of the rank gradient.

Theorem 16. Assume that the multiplicativity of cost-minus-1 problem has an affirmative solution. Let $\left(\Gamma_{n}\right)$ be a Farber chain in $\Gamma$. Then

$$
\operatorname{RG}\left(\Gamma,\left(\Gamma_{n}\right)\right)=\operatorname{RG}(\Gamma) .
$$

In particular, any two Farber chains have the same rank gradient in $\Gamma$.

Proof. Let $\Gamma$ act on a standard Borel probability space $X$ essentially freely. Then using the multiplicativity assumption and Lemma 4, we have

$$
\operatorname{cost}(\Gamma, X)-1=\frac{\operatorname{cost}\left(\Gamma_{n}, X\right)-1}{\left|\Gamma: \Gamma_{n}\right|} \leq \frac{d\left(\Gamma_{n}\right)-1}{\left|\Gamma: \Gamma_{n}\right|},
$$

which implies

$$
\operatorname{cost}(\Gamma, X)-1 \leq \operatorname{RG}\left(\Gamma,\left(\Gamma_{n}\right)\right) .
$$


Now if $\left(\Lambda_{n}\right)$ is another Farber chain in $\Gamma$, then using Theorem 1 and the above inequiality for both chains and actions, we get

$$
\begin{aligned}
\operatorname{RG}\left(\Gamma,\left(\Gamma_{n}\right)\right) & =\operatorname{cost}\left(\Gamma, E\left(\Gamma,\left(\Gamma_{n}\right)\right)\right)-1 \leq \operatorname{RG}\left(\Gamma,\left(\Lambda_{n}\right)\right)=\operatorname{cost}\left(\Gamma, E\left(\Gamma,\left(\Lambda_{n}\right)\right)\right)-1 \\
& \leq \operatorname{RG}\left(\Gamma,\left(\Gamma_{n}\right)\right),
\end{aligned}
$$

so equality holds everywhere.

Let $\varepsilon>0$ and let $H \leq \Gamma$ be a subgroup of finite index such that

$$
\frac{d(H)-1}{|\Gamma: H|}<\operatorname{RG}(\Gamma)+\varepsilon
$$

Using Lemma 10 there exists a Farber chain $\left(\Lambda_{n}\right)$ in $\Gamma$ with $\Lambda_{1}=H$. This implies

$$
\operatorname{RG}\left(\Gamma,\left(\Gamma_{n}\right)\right)=\operatorname{RG}\left(\Gamma,\left(\Lambda_{n}\right)\right) \leq \frac{d(H)-1}{|\Gamma: H|}<\operatorname{RG}(\Gamma)+\varepsilon
$$

and so $\operatorname{RG}\left(\Gamma,\left(\Gamma_{n}\right)\right) \leq \operatorname{RG}(\Gamma)$.

On the other hand, $\operatorname{RG}\left(\Gamma,\left(\Gamma_{n}\right)\right) \geq \operatorname{RG}(\Gamma)$ by definition, so the theorem holds.

Actually, the above argument shows that assuming the multiplicativity of cost-minus-1, a finitely generated residually finite group has fixed price 1 if and only if its absolute rank gradient is 0 .

This could be relevant to decide whether uniform lattices have fixed price 1 . Let $G$ be a semisimple Lie group of $\mathbb{R}$-rank at least 2 and let $\Gamma$ be a lattice in $G$. Gaboriau [11] shows that if $\Gamma$ is nonuniform, i.e., $G / \Gamma$ is not compact, then $\Gamma$ has fixed price 1 . Since, by a theorem of Borel, for every uniform higher rank lattice, there is a nonuniform lattice in the same ambient group, every uniform lattice in $G$ has a measurable action of cost 1 (for the whole argument see [11]). But fixed price is not known for these lattices.

Conjecture 17. All lattices in higher rank Lie groups have absolute rank gradient 0 .

Note that from a result of Sharma and Venkataramana [28], every non-uniform lattice $\Gamma$ in $G$ contains a subgroup of finite index generated by just three elements. This trivially implies $\operatorname{RG}(\Gamma)=0$.

Now we discuss rank 1 lattices. First, by [11], every lattice $\Gamma$ in $\operatorname{SL}(2, \mathbb{R})$ (or $\operatorname{PSL}(2, \mathbb{R})$ ) has fixed price greater than 1 . Using Theorem 1 we deduce that $\operatorname{RG}(\Gamma)>0$ and its value can be computed explicitly from the presentation of the lattice. Next we consider two examples, the first of which have been studied extensively in the literature.

Example A. Let $\Gamma$ be one of the Bianchi groups $\operatorname{SL}\left(2, \mathcal{O}_{d}\right)$, where $\mathcal{O}_{d}$ is the ring of integers of $\mathbb{Q}(\sqrt{-d})$ with $d \in \mathbb{N}$. I. Agol [5] has proved that all the Bianchi groups are virtually free-by-cyclic. From Proposition 13 it follows that $\operatorname{RG}(\Gamma)=0$.

Example B. The lattices from Example A are nonuniform. It is much harder to find uniform lattices of PSL $(2, \mathbb{C})$ with rank gradient zero. One such group has been found by Reid [23, Section 4, Theorem 1]. His example is a uniform arithmetic lattice $\Lambda$ (that is, $\Lambda$ is commensurable with the group of norm 1 elements of a suitable quaternion algebra over a number field $K$ with just one complex embedding). Reid proves that the manifold $\mathbb{H}^{3} / \Lambda$ has a finite cover which fibres over the circle. Group-theoretically this means that 
$\Lambda$ has a subgroup of finite index which is an ascending HNN extension with a finitely generated base group $A$. Proposition 12 now gives that the rank gradient of $\Lambda$ is 0 .

On a question of Kechris and Miller. Example A presents a negative answer to a question of Kechris and Miller [15, Problem 35.7]. They asked whether for a countable infinite Euclidean domain $D$ the group $\operatorname{SL}(2, D)$ has cost 1 if and only if $D$ has infinitely many units. In fact the Bianchi groups have rank gradient 0 and hence cost 1 . We suspect that this is the general behaviour. Indeed, a well-known conjecture by Thurston asserts that every hyperbolic 3-manifold virtually fibres over the circle. This would imply that any lattice in $\operatorname{SL}(2, \mathbb{C})$ has cost 1.

Let $M$ be a finite closed orientable 3-manifold. A Heegaard decomposition (or splitting) for $M$ is an expression of $M$ as a union of two isomorphic handlebodies $h_{1}, h_{2}$ of genus $g \geq 0$ with boundary surfaces $\partial h_{i}$ identified via a homeomorphism $f: \partial h_{1} \rightarrow \partial h_{2}$. Such a decomposition exists by [29, Section 8.3].

The Heegaard genus $g(M)$ of $M$ is the minimal genus $g$ of the surfaces $\partial h_{i}$ in some Heegaard decomposition for $M$. Let $r(M)=d\left(\pi_{1}(M)\right)$ be the rank of $M$.

It is easy to see that the fundamental group $\pi_{1}\left(h_{i}\right)$ surjects onto $\pi_{1}(M)$ and so $g(M) \geq$ $r(M)$. In [32] Waldhausen asked if there is equality. This was proved false for Seifert manifolds by Boileau and Zieschang in [7]. Further work has been done by Schultens and Weidman who construct manifolds $M$ with $g(M)=4 n$ and $r(M) \leq 3 n(n \geq 1)$. However, the question of Waldhausen remained open for hyperbolic 3-manifolds. Also, until now, for all the known counterexamples the ratio $g(M) / r(M)$ was at most $3 / 2$.

We are ready to show that the rank vs Heegaard genus conjecture and fixed price problem conflict with each other.

Proof of Theorem 2. Assume that the fixed price problem has an affirmative solution. Hence, by Lemma 15 and Theorem 16, any Farber chain has rank gradient equal to the absolute rank gradient.

Let us take the group $\Lambda$ in Example B above. Take a chain $\Lambda=N_{0}>N_{1}>\cdots$ of congruence normal subgroups of $\Lambda$ with trivial intersection. Define the manifolds $M_{i}=$ $\mathbb{H}^{3} / N_{i}$. So we have

$$
\pi_{1}\left(M_{i}\right)=N_{i} .
$$

From results of Sarnak and Xue [24] (see also [19, p. 445, Example (f)]) it follows that if $L$ is an arithmetic lattice of $\operatorname{PSL}(2, \mathbb{C})$, then $L$ has property $(\tau)$ with respect to its congruence subgroups. In particular, $\Lambda$ has property $(\tau)$ with respect to the chain $\left\{N_{i}\right\}$ and hence by a recent result of Marc Lackenby [16, Theorem 1.5 and Corollary 1.6] we have

$$
\lim _{i} \frac{g\left(M_{i}\right)}{\left[\Lambda: N_{i}\right]}>0
$$

On the other hand, we have shown that $\operatorname{RG}(\Lambda)=0$, so by Theorem 16 we have

$$
\operatorname{RG}\left(\Lambda,\left(N_{i}\right)\right)=\lim _{i} \frac{r\left(M_{i}\right)}{\left[\Lambda: N_{i}\right]}=0 .
$$

This implies that the ratio $g\left(M_{i}\right) / r\left(M_{i}\right)$ tends to infinity with $i$, thus proving the theorem. 
Remark 1. If the manifold $M$ is noncompact then its canonical compactification $M^{c}$ is a finite 3-manifold with boundary. In this case a Heegaard decomposition of $M$ is its expression as a union of two compression bodies $B_{1}, B_{2}$ with their positive boundaries $\partial B_{i+}$ (both orientable surfaces of genus $g$ ) identified. The boundary of $M$ is the negative boundary $\partial B_{1-} \cup \partial B_{2-}$. See [16, Section 3] for more details. The Heegaard genus $g(M)$ of $M$ is defined to be the minimal genus of the surfaces identified in some decomposition for $M^{c}$ as above. Again it is easy to see that in this case we have the inequality $2 g(M) \geq$ $r(M)$. Assuming the independence of the rank gradient from the chain for the groups in Example A, just as for compact manifolds, the ratio $g(M) / r(M)$ can be arbitrarily large.

Remark 2. Note that one does not need to obtain the multiplicativity of cost-minus-1 for general measurable actions to obtain the independence of rank gradient from the chain. Trivially, it would be enough to settle this for profinite actions. Less trivially, by the recent work of the first author and Weiss [4], it would be enough to settle the multiplicativity for the standard Bernoulli action $\{0,1\}^{\Gamma}$.

Acknowledgments. The authors thank Ian Agol, Gabor Elek, Marc Lackenby and Alan Reid for helpful comments.

\section{References}

[1] Abért, M.: On chains of subgroups in residually finite groups. Preprint

[2] Abért, M., Jaikin-Zapirain, A., Nikolov, N.: The rank gradient from a combinatorial viewpoint. Groups Geom. Dynam. 5, 213-230 (2011) Zbl pre06010056 MR 2782170

[3] Abért, M., Virág, B.: Dimension and randomness in groups acting on rooted trees. J. Amer. Math. Soc. 18, 157-192 (2005) Zbl 1135.20015 MR 2114819

[4] Abért, M., Weiss, B.: Bernoulli actions are weakly contained in any free action. Ergodic Theory Dynam. Systems, to appear

[5] Agol, I.: Criteria for virtual fibering. J. Topology 1, 269-284 (2008) Zbl 1148.57023 MR 2399130

[6] Bergeron, N., Gaboriau, D.: Asymptotique des nombres de Betti, $L^{2}$-invariants et laminations. Comment. Math. Helv. 79, 362-395 (2004) Zbl 1061.55005 MR 2059438

[7] Boileau, M., Zieschang, H.: Heegaard genus of closed orientable Seifert 3-manifolds. Invent. Math. 76, 455-468 (1984) Zbl 0538.57004 MR 0746538

[8] Dooley, A. H., Golodets, V. Ya.: The cost of an equivalence relation is determined by the cost of a finite index subrelation. Preprint

[9] Farber, M.: Geometry of growth: approximation theorems for $L^{2}$ invariants. Math. Ann. 311, 335-375 (1998) Zbl 0911.53026 MR 1625742

[10] Gaboriau, D.: Invariants $l^{2}$ de relations d'équivalence et de groupes. Publ. Math. Inst. Hautes Études Sci. 95, 93-150 (2002) Zbl 1022.37002 MR 1953191

[11] Gaboriau, D.: Coût des relations d'équivalence et des groupes. Invent. Math. 139, 41-98 (2000) Zbl 0939.28012 MR 1728876

[12] Grigorchuk, R. I., Nekrashevich, V. V., Suschanskii, V. I.: Automata, dynamical systems, and groups. Proc. Steklov Inst. Math. 231, 128-203 (2000) Zbl 1155.37311 MR 1841755

[13] Gruschko, I.: Über die Basen eines freien Produktes von Gruppen. Rec. Math. [Mat. Sbornik] N.S. 8 (50), 169-182 (1940) (in Russian; German summary) Zbl 0023.30102 MR 0003412 
[14] Kechris, A. S.: Weak containment in the space of actions of a free group. Israel J. Math. 189, 461-507 (2012) MR 2931406

[15] Kechris, A. S., Miller, B.: Topics in Orbit Equivalence. Lecture Notes in Math. 1852, Springer, Berlin (2004) Zbl 1058.37003 MR 2095154

[16] Lackenby, M.: Heegaard splittings, the virtually Haken conjecture and property $\tau$. Invent. Math. 164, 317-359 (2006) Zbl 1110.57015 MR 2218779

[17] Lackenby, M.: Expanders, rank and graphs of groups. Israel J. Math. 146, 357-370 (2005) Zbl 1066.22008 MR 2151608

[18] Levitt, G.: On the cost of generating an equivalence relation. Ergodic Theory Dynam. Systems 15, 1173-1181 (1995) Zbl 0843.28010 MR 1366313

[19] Lubotzky, A.: Eigenvalues of the Laplacian, the first Betti number and the congruence subgroup problem. Ann. of Math. (2) 144, 441-452 (1996) Zbl 0885.11037 MR 1418904

[20] Lück, W.: $L^{2}$-invariants: Theory and Applications to Geometry and $K$-Theory. Ergeb. Math. Grenzgeb. 44, Springer, Berlin (2002) Zbl 1009.55001 MR 1926649

[21] Neumann, B. H.: On the number of generators of a free product. J. London Math. Soc. 18, 12-20 (1943) Zbl 0028.33901 MR 0008809

[22] Osin, D.: Rank gradient and torsion groups. Bull. London Math. Soc. 43, 10-16 (2011) Zbl pre05853304 MR 2765544

[23] Reid, A.: A non-Haken hyperbolic 3-manifold covered by a surface bundle. Pacific J. Math. 167, 163-182 (1995) Zbl 0817.57014 MR 1318168

[24] Sarnak, P., Xue, X. X.: Bounds for multiplicities of automorphic representations. Duke Math. J. 64, 207-227 (1991) Zbl 0741.22010 MR 1131400

[25] Schlage-Puchta, J.-C.: A p-group with positive rank gradient. J. Group Theory 15, 261-270 (2012)

[26] Schultens, J., Weidman, R.: On the geometric and the algebraic rank of graph manifolds. Pacific J. Math. 231, 481-510 (2007) Zbl 1171.57020 MR 2346507

[27] Shalen, P.: Hyperbolic volume, Heegaard genus and ranks of groups, In: Workshop on Heegaard Splittings (Haifa, Israel, 2005), Geom. Topol. Monogr. 12, Geom. Topol. Publ., Coventry, 335-349 (2007) Zbl 1140.57009 MR 2408254

[28] Sharma, R., Venkataramana, T. N.: Generations of arithmetic groups. Geom. Dedicata 114, 103-146 (2005) Zbl 1112.20044 MR 2174097

[29] Stillwell, J.: Classical Topology and Combinatorial Group Theory. 2nd ed., Springer (1993) Zbl 0774.57002 MR 1211642

[30] Stuck, G., Zimmer, R. J.: Stabilizers for ergodic actions of higher rank semisimple groups. Ann. of Math. (2) 139, 723-747 (1994) Zbl 0836.22018 MR 1283875

[31] Thurston, W.: Geometry and Topology of 3-Manifolds. Lecture notes, Princeton Univ. (1978)

[32] Waldhausen, F.: Some problems on 3-manifolds. In: Proc. Sympos. Pure Math. 32, part 2, Amer. Math. Soc., 313-322 (1978) Zbl 0397.57007 MR 0520549 